\title{
Sharply transitive 1-factorizations of complete multipartite graphs
}

\author{
Giuseppe Mazzuoccolo * and Gloria Rinaldi ${ }^{\dagger}$
}

Submitted: Apr 23, 2009; Accepted: May 17, 2010; Published: May 25, 2010

Mathematics Subject Classification: 05C25; 05C70

\begin{abstract}
Given a finite group $G$ of even order, which graphs $\Gamma$ have a 1 -factorization admitting $G$ as automorphism group with a sharply transitive action on the vertex-set? Starting from this question, we prove some general results and develop an exhaustive analysis when $\Gamma$ is a complete multipartite graph and $G$ is cyclic.
\end{abstract}

\section{Introduction}

A 1-factor of a graph is a collection of edges such that each vertex is incident with exactly one edge. A 1-factorization of a regular graph is a partition of the edge-set of the graph into disjoint 1 -factors. If the graph has valency $v$, then a 1 -factorization is equivalent to a coloring of the edges in $v$ colors (one color for each 1 -factor). The problem of establishing whether a finite simple regular graph $\Gamma$ is 1 -factorizable or not may be an hard task. In fact the 1-factorization problem is NP-complete in general. An obviously necessary condition for the existence of a 1 -factorization is that the number of vertices must be even. So far, the best known sufficient condition is that regular graphs of order $2 n$ and valency $v \geqslant(\sqrt{7}-1) n$ are 1 -factorizable, [6].

For graphs $\Gamma$ with 1 -factorization, then an automorphism group $G$ of the 1 - factorization is a permutation group of the vertex-set of $\Gamma$ which maps 1 -factors onto 1 -factors. The action of $G$ is said to be sharply transitive on the vertex-set if for any given pair of (not necessarily distinct) vertices $x, y$ there exists a unique automorphism $g$ in $G$ mapping $x$ to $y$. Obviously the order of $G$ is equal to the number of vertices in this case. It is well-known that complete graphs are 1 -factorizable and in many recent papers the following question was addressed.

*Dipartimento di Matematica, Università di Modena e Reggio Emilia, via Campi 213/B, Italy. email: mazzuoccolo.giuseppe@unimore.it

${ }^{\dagger}$ Dipartimento di Scienze e Metodi dell'Ingegneria Università di Modena e Reggio Emilia, Via Amendola 2 Padiglione Morselli, I-42100 Reggio Emilia, Italy. email: gloria.rinaldi@unimore.it 
Given a finite group $G$ of even order is it possible to construct a 1-factorization of a complete graph in such a way that $G$ is an automorphism group of the 1-factorization with a sharply transitive action on the vertex set?

A complete answer is not yet available. Paper [8] was the first one presenting the problem and giving a solution for the class of finite cyclic groups (of even order): the answer to the question is negative when the order of the cyclic group is a power of 2 greater than 4 , while it is affirmative for all the other cases. Later on, exhaustive answers were given for other specified classes of groups (for example abelian groups and dihedral groups). We refer to the papers [1], [2], [3], [4], [5], [8], [11] for a complete state of art.

The previous question can be considered as a specified case of the following more general one.

Given a finite group $G$ of even order, which graphs $\Gamma$ have a 1-factorization admitting $G$ as an automorphism group with a sharply transitive action on the vertex set?

First of all, the sharply transitive action of $G$ on the vertex-set, together with the fact that $G$ is a permutation group on the edge-set, forces $\Gamma$ to be a Cayley graph: $\Gamma=C a y(G, \Omega)$. Of course $\Omega$ must be a subset of $G-\left\{1_{G}\right\}$ with the property that $a^{-1} \in \Omega$ for every $a \in \Omega$. ( $1_{G}$ denotes the identity of $G$, and we use in $G$ the multiplicative notation).

If $\Omega=G-\left\{1_{G}\right\}$, then $\Gamma$ is a complete graph and our new question coincides with the original one. Moreover, the graph $\Gamma$ is a complete multipartite graph if and only if $\Omega=G-H$, with $H$ a non trivial subgroup of $G$. The proof of this last statement is quite simple, see Lemma 2.1 of [7] or Proposition 2.2 of [10] for instance.

In this paper we focus our attention on complete multipartite graphs.

We denote a complete multipartite graph with $s$ parts of size $t$ by $K_{s \times t}$. Note that $K_{s \times 1}$ is the complete graph $K_{s}$ and $K_{s \times 2}=K_{2 s}-s K_{2}$, that is, the complete graph $K_{2 s}$ minus the edges of a 1 -factor.

We give an exhaustive answer to our question in the bipartite case. For all other cases, we give an exhaustive answer when the group $G$ is cyclic, except when $s t=2 d, t=2 d^{\prime}$, $d$ and $d^{\prime}$ odd with $d-d^{\prime} \equiv 0 \bmod 4$. In this case the problem is still open and strictly connected with a conjecture presented in [9]. Following [5] we study the problem using the technique of partial differences and the concept of starter.

For the sake of completeness, we observe that the problem of establishing whether a Cayley graph is 1 -factorizable or not is still open. It is conjecture that all Cayley graphs $\operatorname{Cay}(G, \Omega)$ are 1 -factorizable when $\Omega$ generates $G$. There are some partial results on this conjecture, see for example [12], where the conjecture is proved to be true for some classes of groups, in particular when $G$ is cyclic. All graphs studied in this paper are 1-factorizable, but we look for 1-factorizations preserved by $G$.

\section{Preliminary definitions and results}

Let $G$ be a finite group of even order $2 n$ and let $\Omega$ be a subset of $G-\left\{1_{G}\right\}$ with the property that $a^{-1} \in \Omega$ for every $a \in \Omega$. Let $\Gamma=\operatorname{Cay}(G, \Omega)$. Namely the graph with vertex-set $V(\Gamma)=G$ and edge-set $E(\Gamma)=\left\{[x, y] \mid y x^{-1} \in \Omega\right\}$. Obviously $G$ acts on $V(\Gamma)$ by right multiplication. This action is sharply transitive on $V(\Gamma)$ and extends to 
edges and 1-factors. Hence if $R$ is any subset of $V(\Gamma)$ we write: $R g=\{x g \mid x \in R\}$, in particular if $e=[x, y]$ is an edge then $[x, y] g=[x g, y g]$.

The set $\Omega$ is the disjoint union of three sets: $\Omega_{1} \cup \Omega_{2} \cup \Omega_{2}^{-1}$, where $\Omega_{1}$ contains all the involutions of $\Omega$, while $\Omega_{2}$ is defined by the property $h \in \Omega_{2}$ iff $h^{-1} \in \Omega_{2}^{-1}$. The graph $\Gamma$ is given by the orbits under $G$ of the set of edges $\left\{\left[1_{G}, h\right] \mid h \in \Omega_{2}\right\} \cup\left\{\left[1_{G}, j\right] \mid j \in \Omega_{1}\right\}$. In particular $\operatorname{Orb}_{G}\left[1_{G}, j\right]$ with $j \in \Omega_{1}$ has length $n$ and it is a 1 -factor of $\Gamma$. The orbit $\operatorname{Orb}_{G}\left[1_{G}, h\right]$ with $h \in \Omega_{2}$ has length $2 n$ and it is a union of cycles. Namely: if the order of $h$ in $G$ is $t$, then the cycles are given by $\left(x, h x, h^{2} x, \ldots, h^{t-1} x\right)$, as $x$ varies in the set of distinct representatives for the right cosets of the subgroup $<h>$ in $G$. All edges contained in $\bigcup_{h \in \Omega_{2}} \operatorname{Orb}_{G}\left(\left[1_{G}, h\right]\right)$ will be called long edges, while all edges contained in $\bigcup_{j \in \Omega_{1}} \operatorname{Orb}_{G}\left(\left[1_{G}, j\right]\right)$ will be called short edges. Observe that if $[x, y]$ is a short edge of $\Gamma$, then $y x^{-1}$ is necessarily an involution in $\Omega$ and $\operatorname{Stab}_{G}[x, y]=\left\{1_{G}, x^{-1} y\right\}$. We say that $x^{-1} y$ is the involution of $G$ associated with $[x, y]$. If $[x, y]$ is a long edge of $\Gamma$, then $y x^{-1}$ is not an involution in $\Omega$ and $\operatorname{Stab}_{G}[x, y]=\left\{1_{G}\right\}$.

A 1 -factorization of $\Gamma$ which is preserved by the action of $G$ will be called a $G$-regular 1 -factorization. In what follows we ask for the minimum amount of information on the group $G$ and on the set $\Omega$ which is necessary to construct a $G$-regular 1 -factorization of $\Gamma$. Obviously if $\Omega$ is a set of involutions, i.e. $\Omega_{2}=\emptyset$, the graph $\Gamma$ is a set of 1 -factors which constitute all together a $G$-regular 1 -factorization. In this case each 1 -factor is fixed by $G$. For example this happens in each elementary abelian group of even order.

Let $e=[x, y]$ be an edge of $\Gamma$, we define:

$$
\begin{gathered}
\partial([x, y])= \begin{cases}\left\{x y^{-1}, y x^{-1}\right\} & \text { if }[x, y] \text { is long } \\
\left\{x y^{-1}\right\} & \text { if }[x, y] \text { is short }\end{cases} \\
\phi([x, y])= \begin{cases}\{x, y\} & \text { if }[x, y] \text { is long } \\
\{x\} & \text { if }[x, y] \text { is short }\end{cases}
\end{gathered}
$$

If $S$ is a set of edges of $\Gamma$ we define

$$
\partial(S)=\bigcup_{e \in S} \partial(e) \quad \phi(S)=\bigcup_{e \in S} \phi(e)
$$

where, in either case, the union may contain repeated elements and so, in general, will return a multiset.

In the following Definition 1 we generalize the concept of a starter given in [5]. Our definition coincides with the original one if $\Omega=G-\left\{1_{G}\right\}$, i.e., if $\Gamma$ is a complete graph.

If $H$ is a subgroup of $G$ then a system of distinct representatives for the left (right) cosets of $H$ in $G$ will be called a left transversal (right transversal) for $H$ in $G$.

Definition 1 A starter for the pair $(G, \Omega)$ is a set $\Sigma=\left\{S_{1}, \ldots, S_{k}\right\}$ of subsets of $E(\Gamma)$ together with subgroups $H_{1}, \ldots, H_{k}$ of $G$ which satisfy the following conditions:

- $\partial S_{1} \cup \cdots \cup \partial S_{k}$ is a repetition free cover of $\Omega$; 
- for $i=1, \ldots, k$, the set $\phi\left(S_{i}\right)$ is a left transversal for $H_{i}$ in $G$;

- for $i=1, \ldots, k, H_{i}$ must contain the involution associated with any short edge in $S_{i}$.

In the same vein of [5, Theorem 2.2], the following Proposition can be proved.

Proposition 1 There exists a $G$-regular 1 -factorization of $\Gamma=C a y(G, \Omega)$ if and only if there exists a starter for the pair $(G, \Omega)$.

We do not write down the proof which is a simple adaptation of that given in [5]. We just recall that it is constructive and the first bullet in Definition 1 assures that every edge of $\Gamma$ will occur in exactly one $G$-orbit of and edge from $S_{1} \cup \ldots \cup S_{k}$. The second bullet insures that the union of the $H_{i}$-orbits of edges from $S_{i}$ will form a 1-factor. Namely, for each index $i$, we form a 1 -factor as $\cup_{e \in S_{i}} \operatorname{Orb}_{H_{i}}(e)$, whose stabilizer in $G$ is the subgroup $H_{i}$; the $G$-orbit of this 1 -factor, which has length $\left|G: H_{i}\right|$, is then included in the 1 -factorization.

\section{Example.}

Let $G=\mathbb{Z}_{6}$ be the cyclic group in additive notation and let $\Omega=\{1,-1\}$. The graph $\Gamma=\operatorname{Cay}\left(Z_{6}, \Omega\right)$ is the orbit under $\mathbb{Z}_{6}$ of the edge $[0,1]$. A starter is given by $\Sigma=\left\{S_{1}\right\}$ with $S_{1}=\{[0,1]\}$ and associated subgroup $H_{1}=\{0,2,4\}$. We have a $\mathbb{Z}_{6}$-regular 1 factorization of $\Gamma$ with two 1 -factors: $F_{1}=\{[0,1],[2,3],[4,5]\}, F_{2}=\{[1,2],[3,4],[5,0]\}$.

\section{Example.}

Let us denote by $D_{6}$ the dihedral group of order 6 , i.e., the group with defining relations $D_{6}=<a, b \mid a^{6}=b^{2}=1 ; b a=a^{-1} b>$. Let $H=\left\{1, a^{3}, b, b a^{3}\right\}$ and let $\Omega=D_{6}-H$ and consider

$S_{1}=\left\{\left[1, a^{2}\right],\left[a, b a^{2}\right]\right\}, H_{1}=H$

$S_{2}=\{[1, a]\}, H_{2}=\left\{1, a^{2}, a^{4}, b, b a^{2}, b a^{4}\right\}$

$S_{3}=\left\{\left[1, b a^{2}\right]\right\}, H_{3}=D_{6}$,

$S_{4}=\left\{\left[1, b a^{4}\right]\right\}, H_{4}=D_{6}$,

$S_{5}=\left\{\left[1, b a^{5}\right]\right\}, H_{5}=D_{6}$.

The set $\Sigma=\left\{S_{1}, S_{2}, S_{3}, S_{4}, S_{5}\right\}$ together with the subgroups $H_{1}, H_{2}, H_{3}, H_{4}, H_{5}$, is a starter for the pair $(G, \Omega)$. It realizes a $D_{6}$-regular 1 -factorization of $K_{3 \times 4}=\operatorname{Cay}\left(D_{6}, \Omega\right)$.

There are some situations in which the existence of a starter is easily assured. Namely we have the following:

Proposition 2 Let $G$ be a finite group possessing a subgroup $A$ of index 2 and let $J$ be the set of involutions of $G$. Let $\Omega$ be a subset of $(G-A) \cup J$ with the property that $h \in \Omega$ iff $h^{-1} \in \Omega$. Then, there exists a starter for the pair $(G, \Omega)$. 
Proof. Let $\Omega=\Omega_{1} \cup \Omega_{2} \cup \Omega_{2}^{-1}$, where $\Omega_{1}$ contains all the involutions of $\Omega$, while $\Omega_{2}$ is defined by the property $h \in \Omega_{2}$ iff $h^{-1} \in \Omega_{2}^{-1}$. The starter is given by $\Sigma=\left\{S_{h}=\right.$ $\left.\left\{\left[1_{G}, h\right]\right\} \mid h \in \Omega_{1} \cup \Omega_{2}\right\}$. If $h \notin \Omega_{1}$, then $\phi\left(S_{h}\right)$ is a left transversal for $A$ in $G$, while it is a left transversal for the cosets of $G$ itself whenever $h \in \Omega_{1}$.

Proposition 3 Let $G$ be a finite group possessing a subgroup $A$ of index 2 and let $\Sigma^{\prime}=$ $\left\{S_{1}, \ldots, S_{t}\right\}$ be a set of subsets of $E(\Gamma)$ together with subgroups $H_{1}, \ldots, H_{t}$ which satisfy the second and third condition of Definition 1. If $\partial S_{1} \cup \cdots \cup \partial S_{t} \supset A \cap \Omega$ and it does not contain repeated elements, then $\Sigma^{\prime}$ can be completed to a starter for the pair $(G, \Omega)$.

Proof. For each $h \in \Omega$ with $h \notin \partial S_{1} \cup \cdots \cup \partial S_{t}$ we construct the set $S_{h}=\left\{\left[1_{G}, h\right]\right\}$ together with either the subgroup $G$ or $A$ according to whether $h$ is an involution or not, then we adjoin $S_{h}$ to the set $\Sigma^{\prime}$. In this manner we complete $\Sigma^{\prime}$ to a starter for $(G, \Omega)$.

\section{Sharply transitive 1-factorizations of bipartite complete graphs}

We know that the existence of a $G$-regular 1 -factorization of $K_{s \times t}$ necessarily implies the existence of a subgroup of $G$ of order $t$ (see the Introduction and [7], [10]). It will be clear in the next section that this condition is not sufficient to guarantee the existence of a 1-factorization with this property, see Proposition 5. An exception is when the graph is bipartite complete. It is known that bipartite complete graphs are 1-factorizable and we also have the following result.

Proposition 4 There exists a $G$-regular 1 -factorization of $K_{2 \times s}$ if and only if the group $G$ contains a subgroup of index 2 .

Proof. The first part of the proof follows from [7] and [10]. For the second part, let $A$ be a subgroup of index 2 in $G$ and let $\Omega=G-A$. The Cayley graph $\operatorname{Cay}(G, \Omega)$ is $K_{2 \times s}$ and the existence of a $G$-regular 1-factorization follows from Proposition 2.

\section{Cyclic 1-factorizations of multipartite complete graphs}

In this section we focus our attention on the cyclic case. The cyclic group of order $2 n$ will be considered in additive notation and its elements will be the integers between 0 and $2 n-1$, with addition modulo $2 n$. Moreover, when we write down a partial difference $\pm a$, we will always understand $a$ between 0 and the involution $n$ in the natural order of the integers. We will consider multipartite graphs, namely the set $\Omega$ will be of type $Z_{2 n}-H$ with $H$ a suitable proper subgroup of $Z_{2 n}$. To exclude the complete graph, already studied in [8], we will not consider the case $H=<0>$. 


\subsection{A non-existence result}

Proposition 5 Let $G=Z_{2^{m}}$, with d odd and let st $=2^{m} d$. A G-regular 1 -factorization of $K_{s \times t}$ does not exist whenever $t=2^{u} d^{\prime}$ ( $d^{\prime}$ odd) satisfies one of the following conditions:

- $u=m=1$ and $d-d^{\prime} \equiv 2 \bmod 4$;

- $u=1$ and $m>2$.

Proof. Let $G=<1>$ and let $Z_{2^{m}}=\langle d\rangle$ be its subgroup of order $2^{m}$. An element $x \in Z_{2^{m}}$ will be said to be even (respectively odd) if $x=h d, h$ even (resp. odd). Moreover, each element in $G$ can be written uniquely as the sum of an element of $\langle d\rangle$ with an element of $<2^{m}>$, namely $G=<d>\oplus<2^{m}>$. Suppose the existence of a $G$-regular 1 -factorization of $K_{s \times t}$, with $t=2^{u} d^{\prime}, d^{\prime}$ odd, and $u \geqslant 1$. Let $H$ be the subgroup of $G$ of order $t$ and let $\Omega=G-H$. Let $\Sigma=\left\{S_{1}, \ldots, S_{r}\right\}$ be a starter for the pair $(G, \Omega)$. Since $\partial S_{1} \cup \cdots \cup \partial S_{r}=G-H$, the unique involution of $G$ does not appear in this list and then each edge in $S_{1} \cup \cdots \cup S_{r}$ is long. This implies also $\phi\left(S_{i}\right)$ to be of even order for each $i$. Moreover, the set $\phi\left(S_{i}\right)$ is a left transversal for a subgroup $H_{i}$ of $G$ and $2^{m}$ does not divide the order of $H_{i}$, otherwise its index in $G$ would be odd. Therefore we can write uniquely each element of $H_{i}$ as the sum of an element of $K_{i}^{1}$ together with an element of $K_{i}^{2}$, with $K_{i}^{1}$ a suitable subgroup of $\langle d\rangle$ and $K_{i}^{2}$ a suitable subgroup of $\left\langle 2^{m}\right\rangle$, i.e., $H_{i}=K_{i}^{1} \oplus K_{i}^{2}$. Let $e=\left[a_{1}+b_{1}, a_{2}+b_{2}\right]$ be an edge in $S_{i}$ with $a_{1}, a_{2} \in<d>$ and $b_{1}, b_{2} \in<2^{m}>$. We say that $e$ is of type 00 if both $a_{1}$ and $a_{2}$ are even, $e$ is of type 11 if both $a_{1}$ and $a_{2}$ are odd and finally, $e$ is of type 01 if $a_{1}$ and $a_{2}$ are not of the same type. Denote by $x_{i}, y_{i}$ and $z_{i}$ the number of edges in $S_{i}$ which are respectively of type 00,11 and 01 . We obtain $\left|\partial S_{i}\right|=2 x_{i}+2 y_{i}+2 z_{i}$.

Denote by $T_{i}^{1}\left(\right.$ resp. $\left.T_{i}^{2}\right)$ a left transversal for $K_{i}^{1}$ in $\langle d\rangle\left(\right.$ resp. of $K_{i}^{2}$ in $<2^{m}>$ ). The number of even elements in $T_{i}^{1}$ is equal to the number of odd elements of $T_{i}^{1}$, say $t_{i}$. The set $\phi\left(S_{i}\right)$ is a set $R_{i}^{1} \oplus R_{i}^{2}$ which can be obtained by adding elements of the subgroup $K_{i}^{1} \oplus K_{i}^{2}$ to some elements of the set $T_{i}^{1} \oplus T_{i}^{2}$. As $K_{i}^{1} \neq<d>$, no odd element is in $K_{i}^{1}$, moreover, the number of even elements of $R_{i}^{1}$ is $t_{i}$ and it is equal to the number of odd elements in $R_{i}^{1}$. Therefore, $\phi\left(S_{i}\right)$ contains $t_{i}\left|R_{i}^{2}\right|$ even elements and $t_{i}\left|R_{i}^{2}\right|$ odd elements. If $S_{i}$ contains $s \geqslant 0$ edges of type 01 , then the remaining $t_{i}\left|R_{i}^{2}\right|-s$ even elements are paired off to form edges of type 00 in $S_{i}$, as well as the remaining odd elements. We conclude that $x_{i}=y_{i}$ and the number of even elements in $\partial S_{i}$ is divisible by 4 . If $u=m$, then $G-H$ contains $2^{m-1}\left(d-d^{\prime}\right)$ even elements. If $m=1$ and $d-d^{\prime} \equiv 2 \bmod 4$, then this number is not divisible by 4 : we get a contradiction and the first point follows. If $u<m$, then $\left\langle 2^{m-u} d\right\rangle$ is a proper subgroup of $\langle d\rangle$ and it does not contain odd elements. The set $G-H$ contains exactly $2^{m-1} d-2^{u} d^{\prime}$ even elements in this case. If $u=1$ and $m>2$, then this number is not divisible by 4 : we get a contradiction and the second point follows. 


\subsection{An existence result}

Proposition 6 Let $G=Z_{2^{m} d}$, with $d$ odd and let st $=2^{m} d$, with $t=2^{u} d^{\prime}$, $d^{\prime}$ odd. If either $u \neq 1$ or $u=1$ and $m=2$, then a $G$-regular 1 -factorization of $K_{s \times t}$ exists.

Proof. To cover all the possibilities, the proof is divided into 11 cases. The subgroup of $G$ generated by 2 has index 2 and all its elements will be called the even elements of $G$, the other elements of $G$ will be called odd. We will denote by $H$ the subgroup of $G$ of order $2^{u} d^{\prime}$ and we have $K_{s \times t}=\operatorname{Cay}(G, \Omega)$, with $\Omega=G-H$. For each case we will construct a set $\Sigma^{\prime}$ which satisfies the condition of Proposition 3, namely which covers all the even elements of $\Omega$, and then can be completed to a starter. In the first case the construction is explained in details. For the sake of brevity all the other constructions are given, but explanations in details are left to the reader. Pictures and examples will help the reader following the constructions.

- $u=0, m=2, d \neq d^{\prime}, d^{\prime} \equiv 1 \bmod 4$.

We have $G=Z_{4 d}$ and $H=Z_{d^{\prime}}=<h>$ with $h=\frac{4 d}{d^{\prime}}$. Set $\mu=\frac{h}{2}-1$ and $\lambda=\frac{\mu-1}{2}=\frac{h}{4}-1$. The integer $\lambda$ is even, while $\mu$ is odd. To obtain a starter, we construct the following sets of edges:

$B=\{[t, 2 d-2-t] \mid t=0, \ldots, \lambda-1\}$.

For each $k=0, \ldots, \frac{d^{\prime}-3}{2}$, set $A_{k}=\{[\lambda+t+k \mu, 2 d-t-(\lambda+4)-k(\mu+2)] \mid t=0, \ldots, \mu-1\}$. The set $\partial B \cup\left(\cup_{k} \partial A_{k}\right)$ covers all the even elements of $G-H$ except for the involution $2 d$. Moreover, the set $\phi(B) \cup\left(\cup_{k} \phi\left(A_{k}\right)\right)$ covers all the integers from 0 to $2 d-1$ except for the integers: $\lambda+\frac{d^{\prime}-1}{2} \mu ; 2 d-1 ; u_{k}=2 d-(\lambda+2)-k(\mu+2) ; v_{k}=2 d-(\lambda+3)-k(\mu+2)$, with $k=0, \ldots, \frac{d^{\prime}-3}{2}$.

We rearrange these vertices thus obtaining the following edge sets: $C=\left\{\left[2 d-1, u_{0}\right]\right\}, D=\left\{\left[v_{0}, \lambda+\frac{d^{\prime}-1}{2} \mu\right]\right\}, E=\left\{\left[u_{k}, v_{\frac{d^{\prime}-3}{2}-k+1}\right] \mid k=1, \ldots, \frac{d^{\prime}-3}{2}\right\}$. In this manner the set $\phi(B) \cup\left(\cup_{k} \phi\left(A_{k}\right)\right) \cup \phi(C) \cup \phi(D) \cup \phi(E)$ is a set of representatives for the cosets of $Z_{2}$ in $G$. Moreover $\partial C= \pm\{\lambda+1\}, \partial D= \pm\left\{d-1-\frac{d}{d^{\prime}}+\frac{d^{\prime}-1}{2}\right\}$, $\partial E= \pm\left\{1+\left(2 \frac{d}{d^{\prime}}+1\right)\left(\frac{d^{\prime}-1}{2}-2 k\right) \mid k=1, \ldots, \frac{d^{\prime}-3}{2}\right\}$. The set $\partial E \cup \partial C \cup \partial D$ contains pairwise distinct odd differences. They are obviously odd, and we prove that they are pairwise distinct. First of all we prove that $\partial E \cap \partial C=\emptyset$. In fact suppose $\partial E \cap \partial C \neq \emptyset$, and suppose the existence of $\bar{k} \in\left\{1, \ldots, \frac{d^{\prime}-1}{4}-1\right\}$ such that $1+\left(\frac{2 d}{d^{\prime}}+1\right)\left(\frac{d^{\prime}-1}{2}-2 \bar{k}\right)=\frac{d}{d^{\prime}}$. Since $1+\left(\frac{2 d}{d^{\prime}}+1\right)\left(\frac{d^{\prime}-1}{2}-2 \bar{k}\right)$ is between 0 and $2 d$ in this case, the previous equality yields: $1+\frac{d}{d^{\prime}}\left(d^{\prime}-2\right)+\frac{d^{\prime}-1}{2}=\bar{k}\left(2+4 \frac{d}{d^{\prime}}\right)<\left(\frac{d^{\prime}-1}{4}-1\right)\left(2+4 \frac{d}{d^{\prime}}\right)$ which gives the contradiction: $3<\frac{d}{d^{\prime}}-4 \frac{d}{d^{\prime}}$. Now suppose $\bar{k}=\frac{d^{\prime}-1}{4}$ which gives an element of $\partial E$ which is in $\partial D$. We necessarily obtain $\pm 1= \pm \frac{d}{d^{\prime}}$ and again a contradiction.

Finally, if we suppose $\bar{k} \in\left\{\frac{d^{\prime}-1}{4}+1, \ldots, \frac{d^{\prime}-3}{2}\right\}$ with $1+\left(\frac{2 d}{d^{\prime}}+1\right)\left(\frac{d^{\prime}-1}{2}-2 \bar{k}\right)=-\frac{d}{d^{\prime}}$, starting with this equality we obtain:

$1+d+\frac{d^{\prime}-1}{2}=\bar{k}\left(2+4 \frac{d}{d^{\prime}}\right)>\left(\frac{d^{\prime}-1}{4}+1\right)\left(2+4 \frac{d}{d^{\prime}}\right)$ which yields the contradiction: $-1+d>d+3 \frac{d}{d^{\prime}}$. Now we prove that $\partial C \cap \partial D=\emptyset$. In fact at the contrary we should have: $d-1-\frac{d}{d^{\prime}}+\frac{d^{\prime}-1}{2}=$ $\frac{d}{d^{\prime}}$ this yields: $2 \frac{d}{d^{\prime}}+1-\frac{d^{\prime}-1}{2}=d$ which is false, in fact: $d^{\prime}$ is at least 5 and then $2 \frac{d}{d^{\prime}}<d$ and also $1-\frac{d^{\prime}-1}{2}<0$. 
Now we prove that the elements in $\partial E$ are pairwise distinct. Let $k_{1}, k_{2} \in\left\{1, \ldots \frac{d^{\prime}-3}{2}\right\}$, with $k_{1} \neq k_{2}$. The corresponding elements of $\partial E$ obtained from $k_{1}$ and $k_{2}$ are respectively $\pm\left(1+\left(\frac{2 d}{d^{\prime}}+1\right)\left(\frac{d^{\prime}-1}{2}-2 k_{1}\right)\right)$ and $\pm\left(1+\left(\frac{2 d}{d^{\prime}}+1\right)\left(\frac{d^{\prime}-1}{2}-2 k_{2}\right)\right)$. These values are between $-2 d$ and $2 d$. To prove that they are different, it is sufficient to see that both their sum and difference give a non zero element. For the sum we obtain: $2+\left(\frac{2 d}{d^{\prime}}+1\right)\left(d^{\prime}-1-2 k_{1}-2 k_{2}\right)$. If the sum is zero, then $\left(\frac{2 d}{d^{\prime}}+1\right)\left(d^{\prime}-1-2 k_{1}-2 k_{2}\right)=-2$ and this contradicts the following inequality: $\left|\left(\frac{2 d}{d^{\prime}}+1\right)\left(d^{\prime}-1-2 k_{1}-2 k_{2}\right)\right|>3\left|d^{\prime}-1-2 k_{1}-2 k_{2}\right| \geqslant 3$. For the difference we obtain: $2\left(2 \frac{d}{d^{\prime}}+1\right)\left(k_{2}-k_{1}\right)$ which is certainly different from 0 . Finally we prove that $\partial D \cap \partial E=\emptyset$. In fact, we can observe that the elements in $\partial E$ are upper bounded by $1+\left(2 \frac{d}{d^{\prime}}+1\right)\left(\frac{d^{\prime}-5}{2}\right)$ which is certainly less than the positive value $d-1-\frac{d}{d^{\prime}}+\frac{d^{\prime}-1}{2}$ of $\partial D$. The set $\Sigma^{\prime}=\{S\}, S=\left(\cup_{k} A_{k}\right) \cup B \cup C \cup D \cup E$, can be completed to a starter.

In what follows we show an example and the correlated picture:

$G=Z_{60}, H=Z_{5}=<12>, d=15, d^{\prime}=5, m=2, u=0, \mu=5, \lambda=2$.

$B=\{[0,28],[1,27]\}, A_{0}=\{[2,24],[3,23],[4,22],[5,21],[6,20]\}$,

$A_{1}=\{[7,17],[8,16],[9,15],[10,14],[11,13]\}, A=A_{0} \cup A_{1}$,

$E=\{[18,19]\}, C=\{[26,29]\}, D=\{[12,25]\}$,

$S_{1}=A \cup B \cup C \cup D \cup E$.

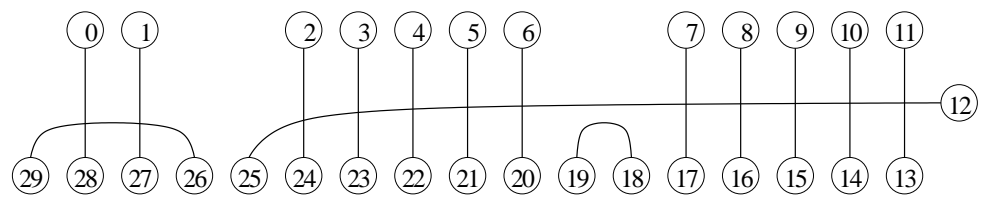

- $u=0, m=2, d \neq d^{\prime}, d^{\prime} \equiv 3 \bmod 4$.

We have $G=Z_{4 d}$ and $H=Z_{d^{\prime}}=<h>$ with $h=\frac{4 d}{d^{\prime}}$.

Set $\mu=\frac{h}{2}-1$ and $\lambda=\frac{\mu-1}{2}=\frac{h}{4}-1$.

Let $k_{1} \in\left\{0, \ldots, \frac{d^{\prime}-3}{4}\right\}$ and $k_{2} \in\left\{0, \ldots, \frac{d^{\prime}-7}{4}\right\}$ (this second set is empty while $d^{\prime}=3$ ) and let $A_{k_{1}}=\left\{\left[\lambda+t+k_{1}(2 \mu+2), 2 d-4-\lambda-t-k_{1}(2 \mu+2)\right] \mid t=0, \ldots \mu-1\right\}$.

$A_{k_{2}}^{\prime}=\left\{\left[\lambda+\mu+2+t+k_{2}(2 \mu+2), 2 d-4-\lambda-\mu-t-k_{2}(2 \mu+2)\right] \mid t=0, \ldots \mu-1\right\}$.

To obtain a starter, we construct the following sets:

$B=\{[t, 2 d-2-t] \mid t=0, \ldots, \lambda-1\}$.

$A=\left(\cup_{k_{1}} A_{k_{1}}\right) \cup\left(\cup_{k_{2}} A_{k_{2}}^{\prime}\right)-\{[d-3, d-1]\}$.

Observe that $\phi(A) \cup \phi(B)$ covers all vertices from 0 to $2 d-1$ except for the following ones: $d-3, d-2, d-1,2 d-1, u_{k_{1}}=2 d-2-\lambda-k_{1}(2 \mu+2), v_{k_{1}}=2 d-2-\lambda-k_{1}(2 \mu+2)-1$, $u_{k_{2}}^{\prime}=\lambda+\mu+k_{2}(2 \mu+2), v_{k_{2}}^{\prime}=\lambda+\mu+k_{2}(2 \mu+2)+1$, with $k_{1} \in\left\{0, \ldots, \frac{d^{\prime}-3}{4}\right\}$ and $k_{2} \in\left\{0, \ldots, \frac{d^{\prime}-7}{4}\right\}$.

Set $d-1=u_{\frac{d^{\prime}+1}{4}}$ and $d-2=v_{\frac{d^{\prime}+1}{4}}$. Rearrange these vertices to construct the following edge sets:

$E=\left\{\left[2 d-1, u_{0}\right],\left[d-3, v_{0}\right]\right\}, C=\left\{\left[u_{r}, v_{\frac{d^{\prime}+1}{4}-r+1}\right] \mid r=1, \ldots, \frac{d^{\prime}+1}{4}\right\}$

$D=\left\{\left[u_{k_{2}}^{\prime}, v_{\frac{d^{\prime}-7}{4}-k_{2}}^{\prime}\right] \mid k_{2}=0, \ldots, \frac{d^{\prime}-7}{4}\right\}$.

Set $S_{1}=A \cup C \cup D \cup E \cup B$. The set $\phi\left(S_{1}\right)$ is a complete system of representatives for the left cosets of $\mathbb{Z}_{2}$ in $G$. The set $\partial A \cup \partial B$ contains distinct elements and covers all the even 
elements of $G-H$ except for the involution $2 d$ and \pm 2 . The set $\partial C \cup \partial D \cup \partial E$ covers some odd distinct elements of $G-H$, the largest of which is $d-\lambda$.

Finally we construct the set $S_{2}=\left\{[0,2 d],[4 d-1,1],[2+t, 3 d-2-t] \mid t=0, \ldots, \frac{d-5}{2}\right\}$. The set $\phi\left(S_{2}\right)$ is a set of representatives for the left cosets of $\mathbb{Z}_{4}$ in $G$ and $\partial S_{2}=\{ \pm 2,2 d\} \cup$ $\{ \pm(d+4), \pm(d+6), \ldots, \pm(2 d-1)\}$. It is possible to verify that the elements of $\partial S_{2}=$ $\{ \pm 2,2 d\} \cup\{ \pm(d+4), \pm(d+6), \ldots, \pm(2 d-1)\}$ are distinct and since the odd differences of $\partial S_{2}$ are greatest or equal to $d+4$, we have $\partial S_{1} \cap \partial S_{2}=\emptyset$. The set $\Sigma^{\prime}=\left\{S_{1}, S_{2}\right\}$ can be completed to a starter.

In what follows we show an example and the correlated picture:

$G=Z_{84}, H=Z_{7}=<12>, d=21, d^{\prime}=7, m=2, u=0, \mu=5, \lambda=2$.

$B=\{[0,40],[1,39]\}, A_{0}=\{[2,36],[3,35],[4,34],[5,33],[6,32]\}$,

$A_{0}^{\prime}=\{[9,31],[10,30],[11,29],[12,28],[13,27]\}$,

$A_{1}=\{[14,24],[15,23],[16,22],[17,21],[18,20]\}, A=A_{0} \cup A_{0}^{\prime} \cup A_{1}-\{[18,20]\}$,

$E=\{[41,38],[18,37]\}, C=\{[26,19],[20,25]\}, D=\{[7,8]\}$,

$S_{1}=A \cup C \cup D \cup E \cup B$.

$S_{2}=\{[0,42],[83,1],[2,61],[3,60],[4,59],[5,58],[6,57],[7,56],[8,55],[9,54],[10,53]\}$
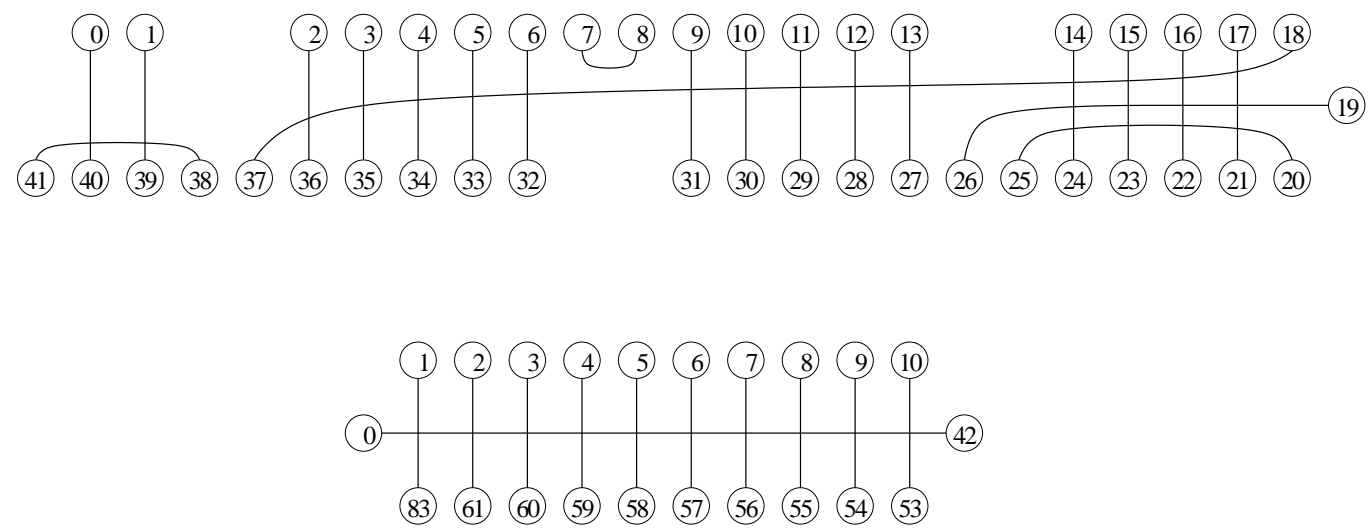

$\partial S_{1}=\{ \pm 40, \pm 38, \pm 34, \pm 32, \pm 30, \pm 28, \pm 26, \pm 22, \pm 20, \pm 18, \pm 16, \pm 14, \pm 10, \pm 8, \pm 6$, $\pm 4, \pm 3, \pm 19, \pm 7, \pm 5, \pm 1\}$.

$\partial S_{2}=\{42, \pm 2, \pm 25, \pm 27, \pm 29, \pm 31, \pm 33, \pm 35, \pm 37, \pm 39, \pm 41\}$.

- $u=0, m=2$ and $d=d^{\prime}$.

We have $G=Z_{4 d}$ and $H=Z_{d}=<4>$. Consider the set $S_{1}=\{[2 s, 2 d-2 s] \mid s=$ $\left.0, \ldots, \frac{d-1}{2}\right\}$ and the subgroup $H_{1}=<d>=Z_{4}$. The set $S_{1}$ contains the short edge [0,2d] and $\phi\left(S_{1}\right)=\left\{0,2 s, d-1+2 s \mid s=1, \ldots, \frac{d-1}{2}\right\}$ is a set of representatives for the cosets of $H_{1}$ in $G$. Furthermore, the set $\partial S_{1}=\left\{2 d, 2 d-4 s, 2 d+4 s \mid s=1, \ldots, \frac{d-1}{2}\right\}$ covers the involution together with all the even elements of $G-H$ (namely all the even elements which are equivalent to 2 modulo 4 ). We conclude that the set $\left\{S_{1}\right\}$ can be completed to a starter.

- $u=0, m=1$ and $d=d^{\prime}$.

$H$ has index 2 in $G$ and the existence of a starter is assured by Proposition 4.

- $u=0, m=1$ and $d \neq d^{\prime}$.

We have $G=Z_{2 d}$ and $H=Z_{d^{\prime}}=<h>$ with $h=\frac{2 d}{d^{\prime}}$. Set $\mu=\frac{h}{2}-1$ and $\lambda=\frac{\mu}{2}$. To obtain a starter, we construct the following sets: 
$B=\{[t, d-1-t] \mid t=0, \ldots, \lambda-1\}$, which contains $\lambda$ edges.

For each $k=0, \ldots, \frac{d^{\prime}-3}{2}$, set $A_{k}=\{[\lambda+k \mu+t, d-\lambda-3-k \mu-2 k-t] \mid t=0, \ldots, \mu-1\}$. Each set $A_{k}$ contains $\mu$ edges.

$C=\left\{\left[\lambda+\frac{d^{\prime}-1}{2} \mu, \lambda+\frac{d^{\prime}-1}{2} \mu+d\right]\right\}$. The set $C$ contains exactly one short edge.

$D=\left\{\left[d-\lambda-1-k(\mu+2), \lambda+\frac{d^{\prime}+1}{2} \mu+1+k(\mu+2)\right] \mid k=0, \ldots, \frac{d^{\prime}-3}{2}\right\}$.

Set $S_{1}=\left(\cup_{k} A_{k}\right) \cup B \cup C \cup D$.

We can prove that $\partial S_{1}$ contains all the distinct even elements of $G-H$, together with some distinct odd elements and the involution (since $\partial C=\{d\}$ ). Moreover $\phi\left(S_{1}\right)$ is a set of representatives for $Z_{2}=<d>$ in $G$. We conclude that the set $\left\{S_{1}\right\}$ can be completed to a starter.

In what follows we write down an example:

$G=Z_{30}, H=Z_{5}=<6>, h=6, \mu=2, \lambda=1$.

$B=\{[0,14]\}, A_{0}=\{[1,11],[2,10]\}, A_{1}=\{[3,7],[4,6]\}$,

$C=\{[5,20]\}, D=\{[8,13],[9,12]\}$.

$\partial S_{1}=\{ \pm 14, \pm 10, \pm 8, \pm 4, \pm 2,15, \pm 5, \pm 3\} . \phi\left(S_{1}\right)=\{0,1, \ldots, 14\}$.

- $u=0, m \geqslant 3, d^{\prime} \equiv 3 \bmod 4$.

Let $G=Z_{2^{m} d}$ and $H=Z_{d^{\prime}}$. Set $h=2^{m} \frac{d}{d^{\prime}}$ and then $H=<h>$, and set $\mu=\frac{h}{2}-1$ and $\lambda=\frac{\mu-1}{2}$. To obtain a starter, we construct the following sets:

$B=\left\{\left[t, 2^{m-1} d-2-t\right] \mid t=0, \ldots, \lambda-1\right\}$, which contains $\lambda$ edges.

Set $A_{k}=\left\{\left[\lambda+k \mu+t, 2^{m-1} d-\lambda-k \mu-4-2 k-t\right] \mid t=0, \ldots, \mu-1\right\}$. For each $k=0, \ldots, \frac{d^{\prime}-3}{2}$. The set $A_{k}$ contains $\mu$ edges.

$C=\left\{\left[\lambda+\frac{d^{\prime}-1}{2} \mu, 2^{m-1} d-1\right]\right\}$.

$D=\left\{\left[2^{m-1} d-2-\lambda-k(\mu+2), \lambda+\frac{d^{\prime}+1}{2} \mu+1+k(\mu+2)\right] \mid k=0, \ldots, \frac{d^{\prime}-3}{2}\right\}$.

Set $S_{1}=\left(\cup_{k} A_{k}\right) \cup B \cup C \cup D$.

We can prove that $\partial S_{1}$ contains all the distinct even elements of $G-H$ except the involution, together with some distinct odd elements. Moreover $\phi\left(S_{1}\right)$ is a set of representatives for $Z_{2}$ in $G$. We conclude that the set $\left\{S_{1}\right\}$ can be completed to a starter.

In what follows we show an example and the correlated picture:

$G=Z_{56}, H=Z_{7}=<8>, h=8, \mu=3, \lambda=1$.

$B=\{[0,26]\}, A_{0}=\{[1,23],[2,22],[3,21]\}, A_{1}=\{[4,18],[5,17],[6,16]\}$,

$A_{2}=\{[7,13],[8,12],[9,11]\}, C=\{[10,27]\}, D=\{[19,20],[14,25],[15,24]\}$.

$\partial S_{1}=\{ \pm 26, \pm 22, \pm 20, \pm 18, \pm 14, \pm 12, \pm 10, \pm 6, \pm 4, \pm 2\} \cup\{ \pm 17, \pm 1, \pm 11, \pm 9\}$.

$\phi\left(S_{1}\right)=\{0,1, \ldots, 27\}$.

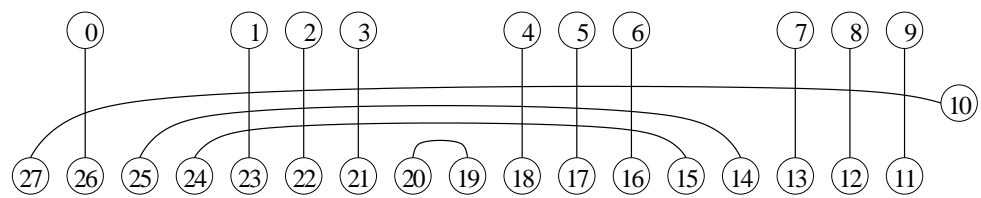

- $u=0, m \geqslant 3, d^{\prime} \equiv 1 \bmod 4$.

Since $d^{\prime}>1$, then it is also $d>1$. Furthermore, we suppose that $H$ is not the trivial group and then $d^{\prime} \geqslant 5$.

We have $G=Z_{2^{m} d}$ and $H=Z_{d^{\prime}}=<h>$ with $h=\frac{2^{m} d}{d^{\prime}}$. 
Set $\mu=\frac{h}{2}-1$ and $\lambda=\frac{\mu-1}{2}=\frac{h}{4}-1$.

Let $k \in\left\{0, \ldots, \frac{d^{\prime}-5}{4}\right\}$ and let

$A_{k}=\left\{\left[\lambda+t+k(2 \mu+2), 2^{m-1} d-4-\lambda-t-k(2 \mu+2)\right] \mid t=0, \ldots \mu-1\right\}$.

$A_{k}^{\prime}=\left\{\left[\lambda+\mu+2+t+k(2 \mu+2), 2^{m-1} d-4-\lambda-\mu-t-k(2 \mu+2)\right] \mid t=0, \ldots \mu-1\right\}$.

To obtain a starter, we construct the following sets:

$B=\left\{\left[t, 2^{m-1} d-2-t\right] \mid t=0, \ldots, \lambda-1\right\}$.

$A=\left(\cup_{k} A_{k}\right) \cup\left(\cup_{k} A_{k}^{\prime}\right)-\left\{\left[2^{m-2} d, 2^{m-2} d-2\right]\right\}$.

Observe that $\phi(A) \cup \phi(B)$ covers all vertices from 0 to $2^{m-1} d-1$ except for the following ones: $2^{m-1} d-1,2^{m-2} d-1,2^{m-2} d-2,2^{m-2} d, \quad u_{k}=2^{m-1} d-2-\lambda-k(2 \mu+2)$,

$v_{k}=2^{m-1} d-2-\lambda-k(2 \mu+2)-1, u_{k}^{\prime}=\lambda+\mu+k(2 \mu+2), v_{k}^{\prime}=\lambda+\mu+k(2 \mu+2)+1$,

with $k \in\left\{0, \ldots, \frac{d^{\prime}-5}{4}\right\}$.

Rearrange these vertices to construct the following edge sets:

$C=\left\{\left[2^{m-1} d-1,2^{m-2} d\right],\left[u_{k}, v_{\frac{d^{\prime}-5}{4}-k}\right] \mid k=0, \ldots, \frac{d^{\prime}-5}{4}\right\}$

$D=\left\{\left[u_{0}^{\prime}, 2^{m-2} d-1\right],\left[v_{0}^{\prime}, 2^{m-2} d-2\right],\left[u_{k}^{\prime}, v_{\frac{d^{\prime}-5}{4}-(k-1)}^{\prime}\right] \mid k=1, \ldots, \frac{d^{\prime}-5}{4}\right\}$.

Set $S_{1}=A \cup C \cup D \cup B$. The set $\phi\left(S_{1}\right)$ is a complete system of representatives for the left cosets of $\mathbb{Z}_{2}$ in $G$. The set $\partial A \cup \partial B$ contains distinct elements and covers all the even elements of $G-H$ except for the involution $2^{m-1} d$ and \pm 2 . The set $\partial C \cup \partial D$ covers some odd distinct elements of $G-H$.

Finally we construct the set $S_{2}=\left\{\left[0,2^{m-1} d\right],\left[2^{m} d-1,1\right],\left[2+t, 2^{m-1} d+d^{\prime}-2-t\right] \mid t=\right.$ $\left.0, \ldots, \frac{d^{\prime}-5}{2}\right\}$. The set $\phi\left(S_{2}\right)$ is a set of representatives for the left cosets of $\mathbb{Z}_{2^{m}}$ in $G$ and $\partial S_{2}=\left\{ \pm 2,2^{m-1} d\right\} \cup\left\{ \pm\left(2^{m-1} d+d^{\prime}-4-2 t\right) \mid t=0, \ldots, \frac{d^{\prime}-5}{2}\right\}=\left\{ \pm 2,2^{m-1} d\right\} \cup\left\{ \pm\left(2^{m-1} d+\right.\right.$ $\left.1+2 t) \mid t=0, \ldots, \frac{d^{\prime}-5}{2}\right\}$. It is possible to verify that the elements of $\partial S_{2}$ are distinct and $\partial S_{1} \cap \partial S_{2}=\emptyset$. Therefore the set $\left\{S_{1}, S_{2}\right\}$ can be completed to a starter.

In what follows we write down an example:

$G=Z_{72}, H=Z_{9}=<8>, \mu=3, \lambda=1$.

$B=\{[0,34]\}, A_{0}=\{[1,31],[2,30],[3,29]\}, A_{0}^{\prime}=\{[6,28],[7,27],[8,26]\}$,

$A_{1}=\{[9,23],[10,22],[11,21]\}, A_{1}^{\prime}=\{[14,20],[15,19],[16,18]\}$

$A=A_{0} \cup A_{0}^{\prime} \cup A_{1} \cup A_{1}^{\prime}-\{[16,18]\}$,

$C=\{[18,35],[24,33],[25,32]\}, D=\{[17,4],[16,5],[12,13]\}$,

$S_{1}=A \cup C \cup D . H_{1}=\mathbb{Z}_{2}$.

$S_{2}=\{[0,36],[71,1],[2,43],[3,42],[4,41]\}$

$\partial S_{1}=\{ \pm 34, \pm 30, \pm 28, \pm 26, \pm 22, \pm 20, \pm 18, \pm 14, \pm 12, \pm 10, \pm 6, \pm 4, \pm 17, \pm 9, \pm 7, \pm 13$, $\pm 11, \pm 1\} . \partial S_{2}=\{36, \pm 2, \pm 41, \pm 39, \pm 37\}$.

- $u \geqslant 2$ and $m>u$ or $u=1$ and $m=2$.

Let $G=Z_{2^{m} d}$ and $H=Z_{2^{u} d^{\prime}}=<h>$. We have $h=2^{m-u} \frac{d}{d^{\prime}}$ and then $H$ does not contain odd elements, moreover the involution $2^{m-1} d$ is in $H$. Set $\mu=\frac{h}{2}-1$. To obtain a starter, we construct the following sets.

For each $k=0, \ldots, 2^{u-1} d^{\prime}-1$, set $A_{k}=\left\{e_{t}^{k} \mid t=0, \ldots, \mu-1\right\}$ with $e_{t}^{k}=\left[t+k \mu, 2^{m-1} d-\right.$ $2-k(\mu+2)-t]$. Observe that $\partial e_{k}^{t}= \pm\left(2^{m-1} d-2-2 t-2 k \mu-2 k\right)$, i.e., it is even and, since $2^{m-1} d-2 k \mu-2 k$ is in $\left\langle h>\right.$, we also have $\partial e_{k}^{t} \equiv \pm 2(-t-1) \bmod h$. As $t$ and $k$ varies, the set $\cup_{k}\left(\partial A_{k}\right)$ covers all the even elements in $G-H$. The set $\cup_{k}\left(\phi\left(A_{k}\right)\right)$ covers all the integers from 0 to $2^{m-1} d-1$ except for the integers: 
$u_{k}=2^{m-1} d-1-(k-1)(\mu+2), v_{k}=2^{m-1} d-k(\mu+2)$, with $k=1, \ldots, 2^{u-1} d^{\prime}$. We rearrange these vertices thus obtaining the following edge set:

$D=\left\{\left[u_{k}, v_{2^{u-1} d^{\prime}-k+1}\right] \mid k=1, \ldots, 2^{u-1} d^{\prime}\right\}$, namely:

$D=\left\{\left[u_{1}, v_{2^{u-1} d^{\prime}}\right],\left[u_{2}, v_{2^{u-1} d^{\prime}-1}\right], \ldots,\left[u_{2^{u-1} d^{\prime}}, v_{1}\right]\right\}$.

In this manner $\cup_{k}\left(\phi\left(A_{k}\right)\right) \cup \phi(D)$ is a set of distinct representatives for the subgroup of order 2 in $G$. Observe also that $\partial D= \pm\left\{1+2(k-1)(\mu+2)-2^{u-1} d^{\prime}(\mu+2) \mid k=\right.$ $\left.1, \ldots, 2^{u-1} d^{\prime}\right\}$. If $u \geqslant 2$, then $\partial D= \pm\left\{1+2\left[(k-1)(\mu+2)-2^{u-2} d^{\prime}(\mu+2) \mid k=1, \ldots, 2^{u-1} d^{\prime}\right\}\right.$ while if $u=1$ and $m=2$ then $\mu$ is even and $\partial D= \pm\left\{1+\left[2(k-1)-d^{\prime}\right](\mu+2) \mid k=\right.$ $\left.1, \ldots, d^{\prime}\right\}$. In both cases $\partial D= \pm\left\{1+2(k-1)(\mu+2)-2^{u-1} d^{\prime}(\mu+2) \mid k=1, \ldots, 2^{u-1} d^{\prime}\right\}$ covers distinct odd elements of $G$. We conclude that the set $S=\left(\cup_{k} A_{k}\right) \cup D$ can be completed to a starter.

In what follows we write down an example: $G=Z_{120}, H=Z_{12}=<10>, d=15, d^{\prime}=3$, $m=3, u=2, \mu=4$.

$A_{0}=\{[0,58],[1,57],[2,56],[3,55]\}, A_{1}=\{[4,52],[5,51],[6,50],[7,49]\}$,

$A_{2}=\{[8,46],[9,45],[10,44],[11,43]\}, A_{3}=\{[12,40],[13,39],[14,38],[15,37]\}$,

$A_{4}=\{[16,34],[17,33],[18,32],[19,31]\}, A_{5}=\{[20,28],[21,27],[22,26],[23,25]\}$,

$D=\{[59,24],[53,30],[47,36],[41,42],[35,48],[29,54]\}$.

$\partial S=\{ \pm 58, \pm 56, \pm 54, \pm 52, \pm 48, \pm 46, \pm 44, \pm 42, \pm 38, \pm 36, \pm 34, \pm 32, \pm 28$,

$\pm 26, \pm 24, \pm 22, \pm 18, \pm 16, \pm 14, \pm 12, \pm 8, \pm 6, \pm 4, \pm 2, \pm 35, \pm 23, \pm 11, \pm 1, \pm 13, \pm 25\}$.

- $m=u=2$.

We have $G=Z_{4 d}$ and $H=Z_{4 d^{\prime}}=<h>$ with $h=\frac{d}{d^{\prime}}$. To obtain a starter, we construct the following sets:

$A_{1}=\left\{[2 t, 2 d-2 t-2] \mid t=0, \ldots, \frac{d-3}{2}\right\}$,

$B_{1}=\left\{[2 d-(2 t+1), 2 d+(2 t+1)] \mid t=0, \ldots, \frac{d-3}{2}\right\}$,

$A_{2}=\left\{[d-2 s h-1, d+2 s h-1] \mid s=1, \ldots, \frac{d^{\prime}-1}{2}\right\}$,

$B_{2}=\left\{[2 d-h(2 s-1), 2 d+h(2 s-1)] \mid s=1, \ldots, \frac{d^{\prime}-1}{2}\right\}$,

$E=\left\{[h(2 s-1), d-2 s h-1] \mid s=1, \ldots, \frac{d^{\prime}-1}{2}\right\}$,

$D=\left\{[d+2 s h-1,2 d-h(2 s+1)] \mid s=0, \ldots, \frac{d^{\prime}-1}{2}\right\}$.

Observe that $A_{2}=\emptyset$ and $B_{2}=\emptyset$ whenever $d^{\prime}=1$. Let $A=A_{1}-A_{2}$ and $B=B_{1}-B_{2}$. The elements of $\partial A$ are equivalent to 0 modulo 4 , while the elements of $\partial B$ are equivalent to 2 modulo 4. Moreover $\partial A \cup \partial B$ covers all the even differences of $G-H$ and $\partial E \cup \partial D$ cover distinct odd differences in $G-H$. In fact, we have $\partial E=\{ \pm(d-4 s h+h-$ 1) $\left.\mid s=1, \ldots, \frac{d^{\prime}-1}{2}\right\}$ and $\partial D=\left\{ \pm(d-4 s h-h+1) \mid s=1, \ldots, \frac{d^{\prime}-1}{2}\right\}$. Observe also that $\phi(E) \cup \phi(D)=\phi\left(B_{2}\right) \cup \phi\left(A_{2}\right) \cup\{d-1, d\}$. We conclude that $\phi(A \cup B \cup E \cup D)$ is a set of distinct representatives for the cosets of $\mathbb{Z}_{2}$ in $G$ and the set $\{S\}, S=A \cup B \cup D \cup E$, can be completed to a starter.

In what follows we write down an example:

$G=Z_{60}, H=Z_{20}=<3>$,

$A_{1}=\{[0,28],[2,26],[4,24],[6,22],[8,20],[10,18],[12,16]\}$,

$A_{2}=\{[2,26],[8,20]\}$,

$B_{1}=\{[29,31],[27,33],[25,35],[23,37],[21,39],[19,41],[17,43]\}$,

$B_{2}=\{[27,33],[21,39]\}, E=\{[3,8],[9,2]\}, D=\{[14,27],[20,21],[26,15]\}$,

$S=\left(A_{1}-A_{2}\right) \cup\left(B_{1}-B_{2}\right) \cup D \cup E$, 
$\partial S=\{ \pm 2, \pm 4, \pm 8, \pm 10, \pm 14, \pm 16, \pm 20, \pm 22, \pm 26, \pm 28, \pm 1, \pm 5, \pm 7, \pm 11, \pm 13\}$.

- $u=m>2$.

We have $G=Z_{2^{m} d}$ and $H=Z_{2^{m} d^{\prime}}=<h>$ with $h=\frac{d}{d^{\prime}}$. Set $\mu=h-1$. To obtain a starter, we construct the following sets:

$D^{\prime}=\left\{u_{r}^{\prime} \mid r=0, \ldots,\left\lceil 2^{m-4} d^{\prime}\right\rceil-1\right\}, D^{\prime \prime}=\left\{u_{s}^{\prime \prime} \mid s=1, \ldots,\left\lceil 2^{m-4} d^{\prime}\right\rceil-1\right\}$,

$E^{\prime}=\left\{v_{r}^{\prime} \mid r=0, \ldots,\left\lceil 2^{m-4} d^{\prime}\right\rceil-1\right\}, E^{\prime \prime}=\left\{v_{s}^{\prime \prime} \mid s=0, \ldots,\left\lceil 2^{m-4} d^{\prime}\right\rceil-2\right\}$ with

$u_{r}^{\prime}=\left[2^{m-2} d+r(2 \mu+2), 2^{m-1} d-1-r(2 \mu+2)\right]$,

$u_{s}^{\prime \prime}=\left[2^{m-2} d-1+s(2 \mu+2), 2^{m-1} d-s(2 \mu+2)\right]$,

$v_{r}^{\prime}=\left[\mu+r(2 \mu+2), 2^{m-2} d-1-\mu-r(2 \mu+2)\right]$,

$v_{s}^{\prime \prime}=\left[\mu+1+s(2 \mu+2), 2^{m-2} d-2-\mu-s(2 \mu+2)\right]$.

For each $k=0, \ldots, 2^{m-3} d^{\prime}-1$, set $A_{k}=\left\{e_{t}^{k}, f_{t}^{k} \mid t=0, \ldots, \mu-1\right\}$ with

$e_{t}^{k}=\left[(2 \mu+2) k+t, 2^{m-1} d-2-k(2 \mu+2)-t\right]$ and

$f_{t}^{k}=\left[\mu+2+k(2 \mu+2)+t, 2^{m-1} d-2-\mu-t-k(2 \mu+2)\right]$.

Set $S_{1}=\left(\cup_{k} A_{k}\right) \cup E^{\prime} \cup E^{\prime} \cup D^{\prime} \cup D^{\prime}$. Recall that $2 \mu+2=2 h$ and observe that $\partial e_{t}^{k}= \pm 2\left(2^{m-2} d-1-k 2 h-t\right)$ and $\partial f_{t}^{k}= \pm 2\left(2^{m-2} d-h-k 2 h-t\right)$. These elements are distinct, even and not contained in $\langle 2 h\rangle$. As $t$ and $k$ varies as prescribed, their number is $4 \mu 2^{m-3} d^{\prime}=2^{m-1} d-2^{m-1} d^{\prime}$ and then, they cover exactly all the even elements in $G-H$. Moreover: $\partial u_{r}^{\prime}= \pm\left(2^{m-2} d-1-4 h r\right), \partial v_{r}^{\prime}= \pm\left(2^{m-2} d-2 h+1-4 h r\right)$, $\partial u_{s}^{\prime \prime}= \pm\left(2^{m-2} d+1-4 h s\right), \partial v_{s}^{\prime \prime}= \pm\left(2^{m-2} d-2 h-1-4 h s\right)$. As $r$ and $s$ varies as prescribed, these elements are distinct, odd and not contained in $\mathrm{H}$.

Finally, $\phi\left(S_{1}\right)$ is a set of representatives for the subgroup $Z_{2}=<2^{m-1} d>$ in $G$. We conclude that the set $\left\{S_{1}\right\}$ can be completed to a starter.

In what follows we write down an example:

$G=Z_{72}, H=Z_{24}=<3>, d=9, d^{\prime}=3, m=3, \mu=2$.

$A_{0}=\{[0,34],[1,33],[4,32],[5,31]\}, A_{1}=\{[6,28],[7,27],[10,26],[11,25]\}$,

$A_{2}=\{[12,22],[13,21],[16,20],[17,19]\}, D^{\prime}=\{[18,35],[24,29]\}, D^{\prime \prime}=\{[23,30]\}$,

$E^{\prime}=\{[2,15],[8,9]\}, E^{\prime \prime}=\{[3,14]\}$.

$\partial S_{1}=\{ \pm 34, \pm 32, \pm 28, \pm 26, \pm 22, \pm 20, \pm 16, \pm 14, \pm 10, \pm 8, \pm 4, \pm 2, \pm 17, \pm 5, \pm 7, \pm 13, \pm 1$, $\pm 11\} . \quad \phi\left(S_{1}\right)=\{0,1, \ldots, 35\}$.

\subsection{Conclusions}

The previous Propositions 5 and 6 cover all the possibilities except for the case of the multipartite graph $K_{s \times t}$, with $s t=2 d, t=2 d^{\prime}, d$ and $d^{\prime}$ odd with $d-d^{\prime} \equiv 0 \bmod 4$. In this case the question is still open. In particular if $d^{\prime}=1$ we have both existence and nonexistence results. In fact, suppose $d^{\prime}=1$, i.e., the subgroup $H=<d>$ is generated by the unique involution of $G$, and let $d \equiv 1 \bmod 4$. If a $G$-regular 1 -factorization of $\operatorname{Cay}(G, G-$ $H)=K_{d \times 2}$ exists, then all edges in $\operatorname{Orb}_{G}[0, d]$ will form a 1-factor for the complete graph $K_{2 d}$. This 1 -factor, together with those of the $G$-regular 1 -factorization of $K_{d \times 2}$ will give rise to a $G$-regular 1 -factorization of $K_{2 d}$ and $\operatorname{Orb}_{G}[0, d]$ will be a 1 -factor fixed by $G$. In [9] the author conjectures the non-existence of such a 1-factorization. In [11] the non-existence is proved when $d$ is a prime, while an example is furnished when $d=21$. 
The situation seems to be more complicated when $d^{\prime}>1$ and to give a complete answer may be a hard task.

\section{References}

[1] Bonisoli, A., Labbate, D., One-Factorizations of Complete Graphs with VertexRegular Automorphism Groups, J. Combin. Des. 10, (2002), 1-16.

[2] Bonisoli, A., Rinaldi, G., Quaternionic Starters, Graphs Combin. 21, (2005), 187-195.

[3] Bonvicini, S., Starters:doubling constructions, Bull. ICA 46, (2006), 88-98.

[4] Bonvicini, S., Frattini based starters in 2-groups, Discrete Math. 308, (2008), 380381.

[5] Buratti, M., Abelian 1-Factorization of the Complete Graph, European J. Combin. 22, (2001), 291-295.

[6] Chetwynd, A.G., Hilton, A.J.W., 1-factorizaing regular graphs of high degree-An improved bound, Discrete Math. 75, (1989), 103-112.

[7] Giudici, M., Li, C.H., Potocnik, P., Praeger, C.E., Homogeneous factorisations of complete multipartite graphs, Discrete Math. 307, (2007), 415-431.

[8] Hartman, A., Rosa, A., Cyclic One-Factorization of the Complete Graph, European J. Combin. 6, (1985), 45-48.

[9] Korchmáros, G., Sharply transitive one-factorizations of the complete graph with an invariant one-factor, J. Combin. Des. 2 (4), (1995), 185-195.

[10] Li, C.H., On isomorphisms of finite Cayley graphs-a survey, Discrete Math. 256, (2002), 301-334.

[11] Rinaldi, G., Nilpotent one-factorizations of the complete graph, J. Combin. Des. , 13 (6), (2005), 393-405.

[12] Stong, A.R., On 1-factorizability of Cayley graphs, J. Combin. Theory Ser. B, 39, (1985), 298-307. 